Support Information

\title{
2D black arsenic phosphorus for ultrafast photonics in near- and mid-infrared regimes
}

Yiqing Shu1, 2, 3, \#, Jia Guo, \#, Taojian Fan², \#, Yijun Xu², Penglai Guo ,

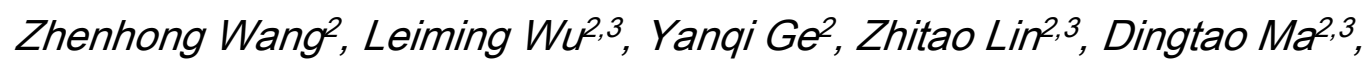
Songrui Wei ${ }^{*}$, Jianqing Li3*, Han Zhang ${ }^{2 *}$, Weicheng Chen ${ }^{\text {* }}$

1School of Physics and Optoelectronic Engineering, Foshan University, Foshan 528000, China

${ }^{2}$ Institute of Microscale Optoelectronics, Collaborative Innovation Centre for Optoelectronic Science \& Technology, Key Laboratory of Optoelectronic Devices and Systems of Ministry of Education and Guangdong Province, College of Physics and Optoelectronic Engineering, Shenzhen Key Laboratory of Micro-Nano Photonic Information Technology, Guangdong Laboratory of Artificial Intelligence and Digital Economy (SZ), Shenzhen University, Shenzhen 518060, PR China

${ }^{3}$ Faculty of Information Technology, Macau University of Science and Technology, Macao 519020, PR China

*Email: chenwch@fosu.edu.cn

\section{CRYSTAL GROWTH}


B-AsP crystals with high quality were synthesized by mineralizer-assisted chemical vapor transport (CVT) method. $500 \mathrm{mg}$ gray arsenic and red phosphorus with molar ratios ranging from 0:10 to 9:1 were used as the precursor. $10 \mathrm{mg}$ tin iodide $\left(\mathrm{Snl}_{4}\right)$ was added as the mineralization agent. The precursor and the mineralization agent were sealed by a Partulab device (MRVS-1002) in a vacuum quartz tube and placed horizontally in the furnace. Firstly, heated the furnace to $750{ }^{\circ} \mathrm{C}$ at a rate of $5{ }^{\circ} \mathrm{C} / \mathrm{min}$ and holding at this temperature for two hours. Then cooled the furnace to $500{ }^{\circ} \mathrm{C}$ within 7.5 hours and holding for another 3 hours at this temperature. After that, the furnace was slowly cooled to $150{ }^{\circ} \mathrm{C}$ in 8 hours, and then gradually cooled to room temperature. Finally, b-AsP crystals can be found on the cold end of the tube.

\section{PREPARATION AND CHARACTERIZATION OF 2D b-AsP NANOSHEETS}

We successfully prepared 2D b-AsP nanosheets by LPE method. As shown in Figure 1(a), isopropyl alcohol (IPA) was selected as stripping solvent because of its high stripping efficiency and experimental safety. Put $100 \mathrm{mg}$ of bulk bAsP and a small amount of IPA solvent into the agate mortar and grind for 3060 minutes to ensure that the bulk is ground into small pieces. Next, the bAsP/IPA slurry was transported to a $25 \mathrm{~mL}$ plastic tube, and an additional 15 $\mathrm{mL}$ IPA solvent was added to dilute the slurry to $0.2 \mathrm{mg} / \mathrm{mL}$ Under the protection of Ar atmosphere, typical probe sonication was carried out for $10 \mathrm{~h}$ with a power of $200 \mathrm{~W}$. After the probe sonication, the b-AsP/IPA mixture was further treated with bath sonication at $400 \mathrm{~W}$ for $10 \mathrm{~h}$. During the probe sonication and bath 
sonication, the mixture is kept below $10^{\circ} \mathrm{C}$ to avoid oxidation of the sample. At last, the ideal sizes of 2D b-AsP nanosheets were obtained by gradient centrifugation method.

\section{CHARACTERIZATION}

SEM (Carl Zeiss, Supra 55 Sapphire) and TEM (FEI Tecnai G2 Spirit, 80 kV) were used to characterize the morphology of 2D b-AsP Nanosheets. AFM (Bruker, Dimension ICON, with 512 pixels per line) was used to evaluate the thickness of 2D b-AsP Nanosheet. The sample for AFM measure was prepared by dropping 2D b-AsP/IPA mixture onto $\mathrm{Si} / \mathrm{SiO}_{2}$ substrates. Optical absorption spectra of 2D b-AsP nanosheets were measured using a UV-Vis-NIR spectrometer (Cary 60, Agilent). A high-resolution confocal Raman microscope (HORIBA, LabRAM, HR800) was used to determine the Raman spectrum of 2D b-AsP at room temperature. The chemical compositions of b-AsP were analyzed by an X-ray photoelectron spectroscopy (XPS, Thermo Scientific, ESCALAB 250Xi).

\section{Z-SCAN}

A mode-locked Ti:Sapphire laser is operating at $800 \mathrm{~nm}$ to output femtosecond pulses and an optical parametric amplifier (OPA, TOPAS prime, SpectraPhysics) system is used to amplify femtosecond pulses. The pulse duration of the laser is $100 \mathrm{fs}$ and repetition rate is $1 \mathrm{kHz}$. The sample moves along the optical axis under the control of the computer electronic platform and passes through the focus of the focusing lens. An infrared (918-IR-OD3R, NEWPORT) 
photodetector was used to record total transmittances of the sample versus with the incident laser intensity.

\section{ALL-FIBER LASER SYSTEMS}

EDF and TDF lasers have similar ring structures including a pump source, a wavelength division multiplexer (WDM), a segment of gain fiber, an output coupler (OC), a polarization independent isolator (PI-ISO), a polarization controller (PC) and a 2D b-AsP SA. But the devices for composing the lasers are individually different. First, a pump source with a wavelength of $976 \mathrm{~nm}$ connected with a 980/1550 single-mode WDM were used for EDF laser, while $1570 \mathrm{~nm}$ pump source connected with a 1570/2000 single-mode WDM was used for TDF laser. Second, EDF with dispersion parameters of $61.11 \mathrm{ps}^{2} \mathrm{~km}^{-}$ ${ }^{1}$ and $0.8 \mathrm{~m}$ length, and TDF with dispersion parameters of $-71 \mathrm{ps}^{2} \mathrm{~km}^{-1}$ and 2.7 $\mathrm{m}$ length were used as gain medium in EDF and TDF laser cavity, respectively. Thirdly, PC winding fiber in EDF laser and TDF laser are SMF-28e and SM1950, respectively. And last, a 90/10 OC was used for the output port of the EDF laser, while an 80/20 OC was used for that of the TDF laser. These output laser signals are detected by an optical spectrum analyzer (Yokogawa AQ6370D for EDF laser and Yokogawa AQ6375B for TDF laser), an oscilloscope (KEYSIGHT DSOS104A for EDF laser, Tektronix MD04104C for TDF laser), a RF spectrometer (Agilent N9030A for EDF laser and Keysight N9322C for TDF laser) and an autocorrelator (APE-150 Pulsecheck).

\section{6. $L_{\text {eff }}$ On 2D b-AsP at each wavelength.}




\begin{tabular}{lll}
\hline$\lambda(\mathrm{nm})$ & $\mathrm{A}$ & $\mathrm{L}_{\text {eff }}(\mu \mathrm{m})$ \\
\hline 800 & 0.733 & 119.64 \\
1550 & 0.355 & 168.91 \\
1800 & 0.298 & 178.29 \\
\hline
\end{tabular}

Table S1 Different values of $L_{\text {eff }}$ on 2D b-AsP at each wavelength.

\section{Crystal structure.}

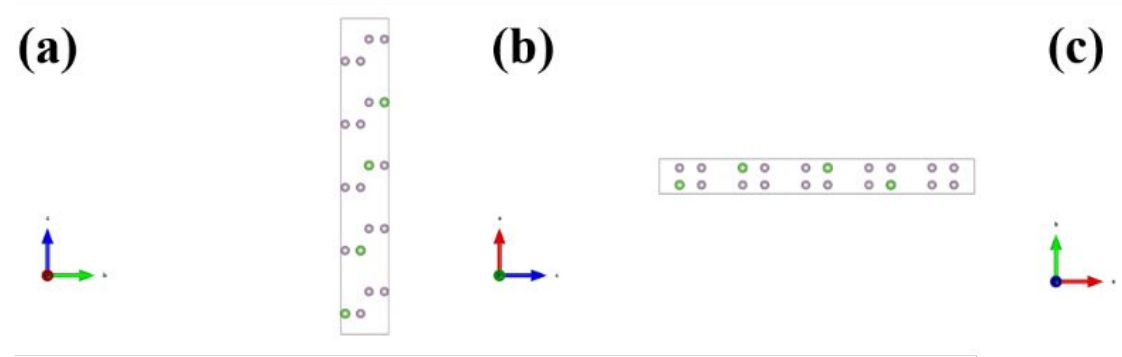

Figure S1 Crystal structure of a: (1 15) case.

(a)

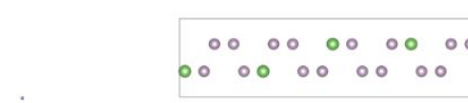

$\stackrel{\jmath}{\hookrightarrow}$ (b)

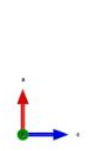

(c)

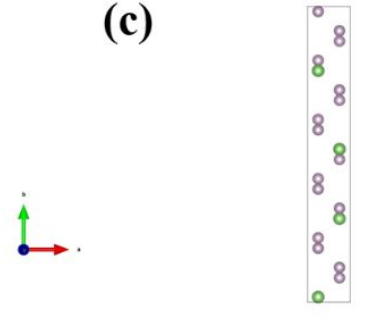

Figure S2 Crystal structure of b: $\left(\begin{array}{lll}5 & 1 & 1\end{array}\right)$ case.

(a)

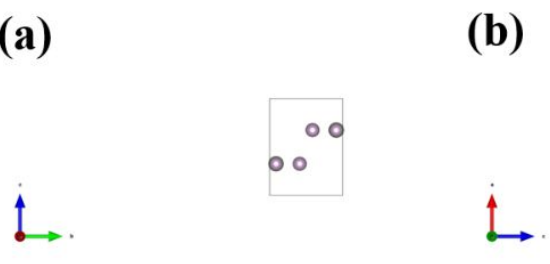

(b)

Figure S3 Crystal structure of c: (1 51 1) case. 